\title{
Studies of fission fragment properties at the Los Alamos Neutron Science Center (LANSCE)
}

\author{
Fredrik Tovesson ${ }^{\mathrm{a}}$, Dmitriy Mayorov, Dana Duke, Brett Manning, and Verena Geppert-Kleinrath \\ Los Alamos National Laboratory, PO Box 1663, Los Alamos, NM 87545, USA
}

\begin{abstract}
Nuclear data related to the fission process are needed for a wide variety of research areas, including fundamental science, nuclear energy and non-proliferation. While some of the relevant data have been measured to the required accuracies there are still many aspects of fission that need further investigation. One such aspect is how Total Kinetic Energy (TKE), fragment yields, angular distributions and other fission observables depend on excitation energy of the fissioning system. Another question is the correlation between mass, charge and energy of fission fragments. At the Los Alamos Neutron Science Center (LANSCE) we are studying neutron-induced fission at incident energies from thermal up to hundreds of $\mathrm{MeV}$ using the Lujan Center and Weapons Neutron Research (WNR) facilities. Advanced instruments such as SPIDER (timeof-flight and kinetic energy spectrometer), the NIFFTE Time Projection Chamber (TPC), and Frisch grid Ionization Chambers (FGIC) are used to investigate the properties of fission fragments, and some important results for the major actinides have been obtained.
\end{abstract}

\section{Introduction}

Over the last decade we have seen a resurgence in fission research, both in terms of experimental capabilities and new theoretical advances. There are a number of reasons for the renewed interest in this nuclear reaction process that was discovered more than 75 years ago, and one of the driving factors is a need for more accurate nuclear data for science and technology. In nuclear technologies we need to accurately understand and characterize criticality, and the nuclear fission data has the most impact on criticality. Properties of fission are also important to nuclear technologies in a different way; fission products and radiation output can be used to diagnose and characterize nuclear performance. One such example is the practice to determine the presence of the ${ }^{148} \mathrm{Nd}$ fission product in spent nuclear fuel to determine the burn-up level. There are currently several experimental and theoretical efforts underway at Los Alamos National Laboratory (LANL) to advance our understanding of the fission process; here we will describe the experimental program to measure properties of fission fragments.

\section{Experimental method}

The Los Alamos Neutron Science Center (LANSCE) [1] is a powerful neutron source that allows us to study nuclear reaction from thermal energies up to several hundreds of $\mathrm{MeV}$. Fission fragment properties are currently studied using three different instruments: a fission fragment timeof-flight and kinetic energy spectrometer (SPIDER), a Time Projection Chamber (TPC), and a double Frisch grid ionization chamber.

\footnotetext{
a e-mail: tovesson@lanl.gov
}

\subsection{Neutron source}

Two facilities at LANSCE are used for fission fragment studies: Weapons Neutron Research (WNR) and the Lujan Center (see Fig. 1). At both facilities neutrons are produced when the $800-\mathrm{MeV}$ proton beam from the LANSCE accelerator impinges on tungsten spallation targets. The beam is pulsed, such that the neutron time-of-flight method can be applied to make energy-differential measurements. WNR has a bare spallation target that produces fast neutrons, while the Lujan Center target is surrounded by moderators to achieve thermalized neutrons. The flight paths used for fission experiments varies from 6 up to $25 \mathrm{~m}$. At WNR the proton pulse spacing $(1.8 \mu \mathrm{s})$ and pulse width (about $250 \mathrm{ps}$ ) enable measurements for neutron energies from a few hundred $\mathrm{keV}$ to hundreds of $\mathrm{MeV}$.

\subsection{Spectrometer for fission fragment determination in fission research (SPIDER)}

The SPIDER instrument is a type of spectrometer that measures the velocities and kinetic energies of fission fragments in order to determine their mass. SPIDER has two identical collinear spectrometers that allows for coincidental measurements of the two complementary fragments typically emitted in fission. The time-of-flight (TOF) section used to determine the fission fragments' velocities consists of a start- and a stop-timing pick-off detector separated by about $70 \mathrm{~cm}$. The TOF section is kept at high vacuum, and a detailed description of the setup and performance can be found in Ref. [2]. After the TOF section, the fragments pass through a thin $(200 \mathrm{~nm})$ $\mathrm{SiN}_{4}$ window to enter into an ionization chamber where the kinetic energy is registered. The full system was benchmarked using a ${ }^{252} \mathrm{Cf}$ spontaneous fission source, and the results can be found in Ref. [3]. 


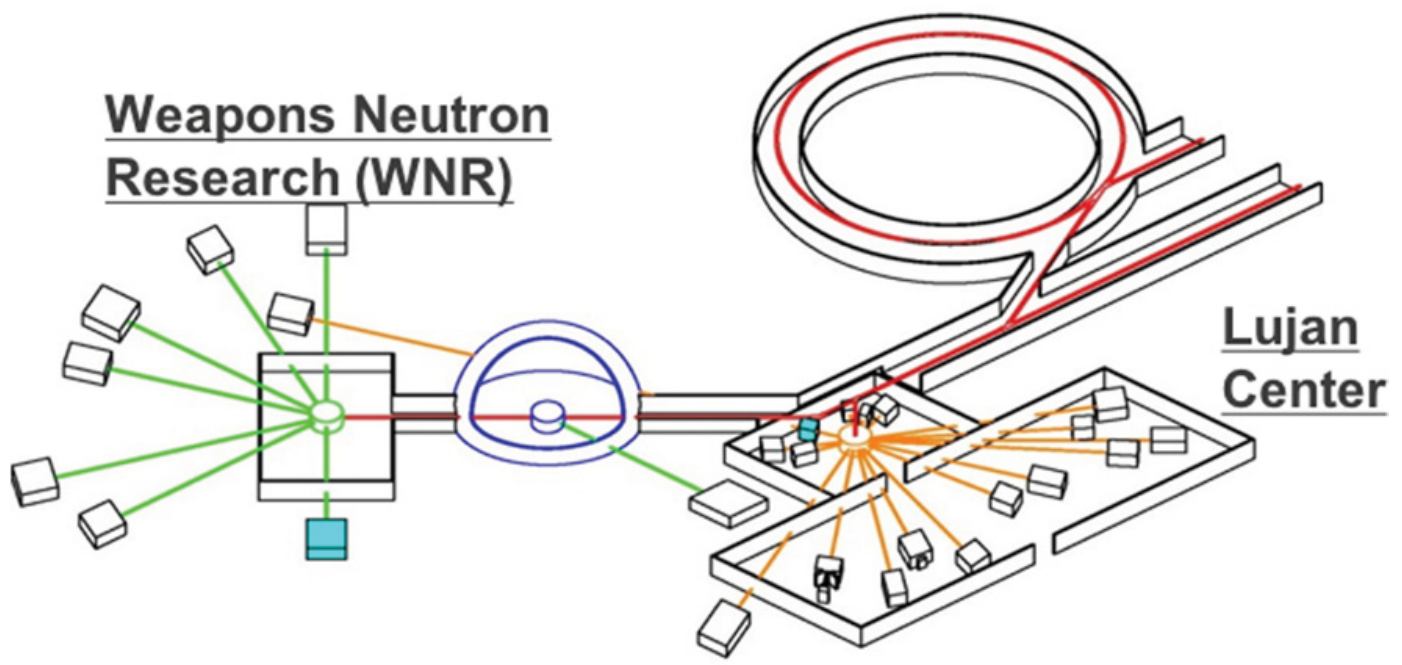

Figure 1. Schematic view of the Lujan Center and WNR facilities at LANSCE.

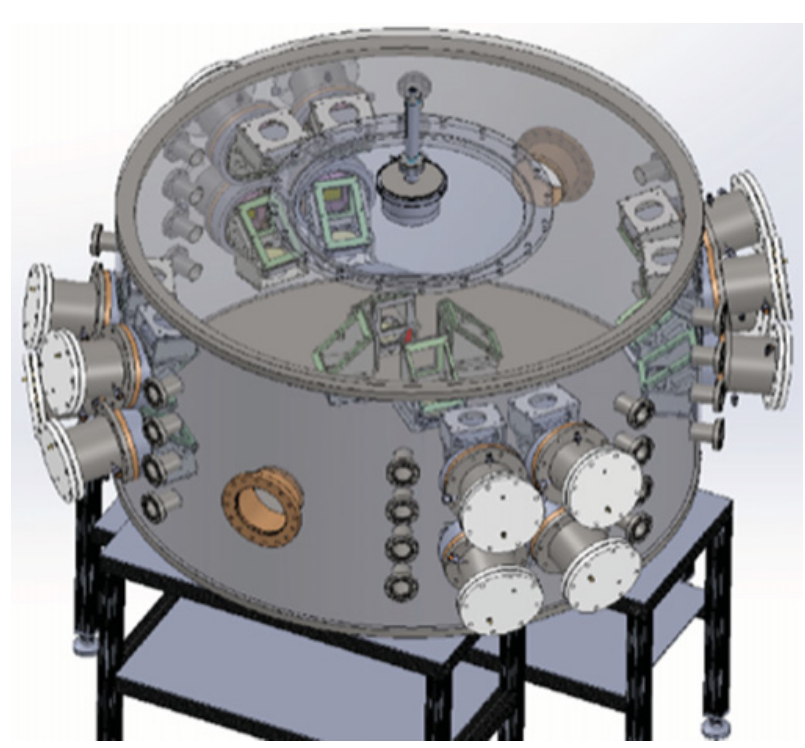

Figure 2. Mechanical drawing of the MegaSPIDER instrument.

The main limitation of the SPIDER instrument is the low detection efficiency stemming from a small coverage of the solid angle $(<0.1 \%)$. In order to study fission induced by fast neutrons a new version of SPIDER, MegaSPIDER, is under construction. This instrument will have 16 individual spectrometer "arms" in order to increase the efficiency. A drawing of MegaSPIDER in show in Fig. 2.

\subsection{Frisch grid ionization chambers}

Frisch grid ionization chambers are used for fission fragment studies at LANSCE as a complementary technique to SPIDER. While these detectors provide lower mass resolution (4-5 amu FWHM) than the SPIDER instrument, they have the advantage of covering almost the full solid angle, resulting in about $95 \%$ detection efficiency. This makes them ideal to study changes in fission fragment properties as a function of incident neutron energies with fine binning. This detector [4] type has been used to measure the total kinetic energy release (TKE) and mass yields in fission of various actinides.

The Frisch grid ionization chambers consist of a gas volume with a central cathode where the fissionable target is mounted. On each side is an anode and a grid that shields the anode. The kinetic energies of the two fission fragments are measured by the two individual sections of the chamber. A detailed description of Frisch grid ionization chambers and how they can be used to determine mass yields can be found in Ref. [5].

\subsection{The NIFFTE Time Projection Chamber (TPC)}

The Neutron Induced Fission Fragment Tracking Experiment (NIFFTE) collaboration has developed a Time Projection Chamber (TPC) with the goal of measuring fission cross sections with unprecedented accuracy. This is a particle tracking detector with almost 6000 channels that are used to detect fission fragments. The uncertainty in cross section measurements is reduced with this instrument compared to conventional fission chambers since it allows for in situ radiographs of the samples and beam imaging. It also provides better particle identification and superior determination of detection efficiency. A detailed description of the fission TPC can be found in Ref. [6].

\section{Results and conclusions}

Properties of fission fragments have been studied for several actinides at LANSCE, including ${ }^{235} \mathrm{U},{ }^{238} \mathrm{U}$, and ${ }^{239} \mathrm{Pu}$. The measurements have been performed at both fast and thermal neutron energies using the Lujan Center and WNR facilities at LANSCE.

The average total kinetic energy $(\overline{T K E})$ has been studied as a function of incident neutron energy in an effort to provide more reliable data, particularly around $14 \mathrm{MeV}$ of incident neutron energy. Historically, the ENDF/B nuclear data library that is used for nuclear science and technology would provide one value for $\overline{T K E}$ for each of the major actinides, independent of incident neutron energy. However, as discussed by D. Madland in his 2006 paper [7], experimental evidence shows that $\overline{T K E}$ tend to get lower as the incident neutron energy increases. 


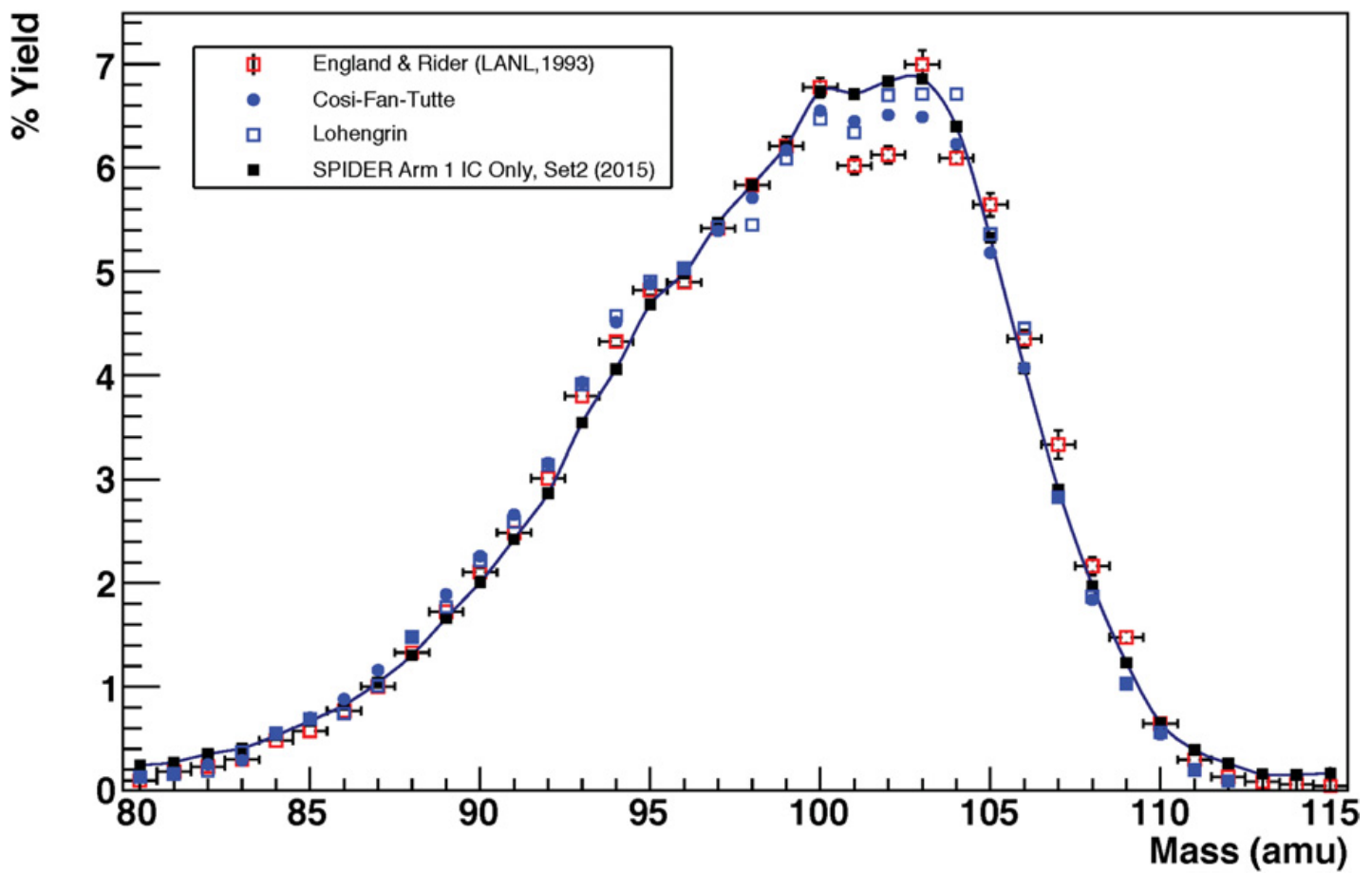

Figure 3. Preliminary mass yields for light fission fragments from ${ }^{239} \mathrm{Pu}\left(\mathrm{n}, \mathrm{f}_{t h}\right)$ measured with the SPIDER instrument. Also shown is an evaluation and other experimental data from literature.

The Madland paper provided fits to experimental data, but the author made clear that the fit was only valid up to $\mathrm{E}_{n}=9 \mathrm{MeV}$. However, those fits were used to extrapolate to $14 \mathrm{MeV}$ and the resulting values were included in ENDF. The purpose of the new measurements is to address this issue, and ensure that $\overline{T K E}$ is well characterized at all energies relevant to applications. The results for ${ }^{238} \mathrm{U}$ can be found in Ref. [8] and for ${ }^{239} \mathrm{Pu}$ in Ref. [9].

The current issue with fission product yields is also related to changes as a function of incident neutron energy. Specific fission products such as ${ }^{148} \mathrm{Nd}$ are commonly used to determine the number of fission reactions in nuclear material, and even relatively small yield changes versus neutron energy are therefore important. The general trends in the mass yield distributions have been measured with Frisch grid chambers, and the results for ${ }^{238} \mathrm{U}$ are published in Ref. [8]. The SPIDER instrument provides high mass resolution and is being used to deduce individual fission product yields, but so far only thermal neutron measurements have been performed. A preliminary result for the light mass peak for ${ }^{239} \mathrm{Pu}$ is shown in Fig. 3. The changes in yield for fast neutrons will be studied with the MegaSPIDER instrument in the future.

The high-precision fission cross section measurements with the Time Projection Chamber (TPC) are ongoing. There are also other aspects of fission that are being studied with the TPC, such as the fission fragment angular distribution anisotropy. The anisotropy in fission of ${ }^{235} \mathrm{U}$ has been determined and is available in a Ph.D. thesis by Verena Geppert-Kleinrath [10].

With this suite of different instruments and neutrons sources available for fission research (both the ones described here and others world-wide), combined with other approaches such as inverse-kinematics studies, we have an opportunity to significantly advance and refine our understanding of fission in the coming years. This is indeed an interesting time for the field, and the advances made will benefit both science and technology.

\section{References}

[1] P.W. Lisowski, K.F. Schoenberg, Nucl. Instr. and Meth. A 562, 910 (2006)

[2] C.W. Arnold, F. Tovesson, K. Meierbachtol, T. Bredeweg, M. Jandel, H.J. Jorgenson, A. Laptev, G. Rusev, D. Shields, M. White, A.A. Hecht, R.E. Blakeley, D.M. Mader, Nucl. Instr. and Meth. A 764, 53 (2014)

[3] K. Meierbachtol, F. Tovesson, D. Shields, C. Arnold, R. Blakeley, T. Bredeweg, M. Devlin, A.A. Hecht, L.E. Heffern, J. Jorgenson, A. Laptev, D. Mader, J.M. O’Donnell, A. Sierk, M. White, Nucl. Instr. and Meth. A 788, 59 (2015)

[4] S. Mosby, F. Tovesson, A. Couture, D. Duke, V. Kleinrath, R. Meharchand, K. Meierbachtol, J.M. O’Donnell, B. Perdue, D. Richman, D. Shields, Nucl. Instr. and Meth. A 757, 75 (2014)

[5] C. Budtz-Jorgensen and H.-H. Knitter and Ch. Straede and F.-J. Hambsch and R. Vogt, Nucl. Instr. and Meth. A 759, 50 (2014)

[6] M. Heffner, D.M. Asner, R.G. Baker, et al., Nucl. Instr. and Meth. A 258, 209 (1987)

[7] D.G. Madland, Nucl. Phys. A 772, 113 (2006)

[8] D.L. Duke, F. Tovesson, A.B. Laptev, S. Mosby, F.-J. Hambsch, T. Brys, and M. Vidali, Phys. Rev. C 94, 054604 (2016)

[9] K. Meierbachtol, F. Tovesson, D.L. Duke, V. Kleinrath, B. Manning, R. Meharchand, S. Mosby, and D. Shields, Phys. Rev. C 94, 034611 (2016)

[10] V. Kleinrath, PhD dissertation, Technische Universität Wien, 2016 